\title{
Autonomous configuration of parameters in robotic digital cameras
}

\author{
António J. R. Neves, Bernardo Cunha, Armando J. Pinho and Ivo Pinheiro \\ Signal Processing Lab, DETI / IEETA \\ University of Aveiro, 3810-193 Aveiro, Portugal \\ an@ua.pt / mbc@det.ua.pt / ap@ua.pt / pinheiro@ua.pt
}

\begin{abstract}
In the past few years, the use of digital cameras in robotic applications has been increasing significantly. The main areas of application of these robots are the industry and military, where these cameras are used as sensors that allow the robot to take the relevant information of the surrounding environment and making decisions. To extract information from the acquired image, such as shapes or colors, the configuration of the camera parameters, such as exposure, gain, brightness or white-balance, is very important. In this paper, we propose an algorithm for the autonomous setup of the most important parameters of digital cameras for robotic applications. The proposed algorithm uses the intensity histogram of the images and a black and a white area, known in advance, to estimate the parameters of the camera. We present experimental results that show the effectiveness of our algorithms. The images acquired after calibration show good properties for further processing, independently of the initial configuration of the camera.
\end{abstract}

\section{Introduction}

Vision is an extremely important sense for both humans and robots, providing detailed information about the environment. A robust vision system should be able to detect objects reliably and present an accurate representation of the world to higher-level processes, not only under ideal conditions, but also under changing lighting intensity and color balance.

To extract information from the acquired image, such as shapes or colors, the camera calibration procedure is very important. If the parameters of the camera are wrongly calibrated, the image details are lost and it may become almost impossible to recognize anything based on shape or color.

The work that we present in this paper ${ }^{1}$ is related to the Middle Size League (MSL) of Robocup. The MSL competition of RoboCup is a standard real-world test for autonomous multi-robot control. The ultimate goal of the RoboCup project is, by 2050, to develop a team of fully autonomous humanoid robots that can win against the human world champion soccer team. It means that,

\footnotetext{
${ }^{1}$ This work was partially supported by project ACORD (Adaptive Coordination of Robotic Teams), FCT/PTDC/EIA/70695/2006.
} 
in a near future, the robots will have to play under natural lighting conditions and in outdoor fields. This introduces many obstacles to the robots because they must be able to play either under controlled lighting conditions, as is the case of artificial illumination, as well as in non-controlled lighting conditions, such as in outdoor fields. In outdoor fields the illumination can change slowly during the day, due to the movement of the sun, as well as fast in short periods of time due to a partial and temporally covering of the sun by clouds. Consequently, the robots have to adjust, in real time, its color segmentation values as well as its camera parameters according to the lighting conditions [1].

As far as we can understand from the published work made by the other teams of RoboCup MSL, most of them don't have any software in the robots to autonomous calibrate the most important parameters of the digital cameras, which means that their cameras are only adjusted manually at the beginning of each game. Some of the teams have tried to solve the problem by developing algorithms for run-time color calibration (see for example [2]).

In this work, we show that the problem can be solved by adjusting the parameters of the camera in order to guarantee the correct colors of the objects, allowing the use of the same color classification independently of the light conditions. In the team description papers of Brainstormers Tribots [3], NuBot [4], Tech United [5] and Robofoot PM [6], some auto-calibration algorithms of the camera parameters are mentioned, although they don't present any details about them.

We propose an algorithm to configure the most important parameters of the cameras, namely exposure, white-balance, gain and brightness without human interaction. We use the histogram of intensities of the images acquired and a black and a white area, known in advance, to estimate the referred parameters of the camera. This approach differs from the well known problem of photometric camera calibration (a survey can be found in [7]), since we are not interested in obtaining the camera response values but only to configure its parameters according to some measures obtained from the acquired images in robotic applications. The self-calibration process for a single robot requires a few seconds, including the time necessary to interact with the application, which is considered fast in comparison to the several minutes needed for manual calibration by an expert user.

The algorithms proposed in this paper have been tested in the CAMBADA MSL soccer team of the University of Aveiro. The general architecture of the CAMBADA robots has been described in [8]. The vision system of the CAMBADA robots is based on an hybrid vision system, formed by an omnidirectional vision sub-system (Point Grey Flea 2 camera) and a perspective vision sub-system (Unibrain Fire-i camera), that together can analyze the environment around the robots, both at close and long distances [9]. 


\section{Configuration of the camera parameters}

The configuration of the parameters of digital cameras is crucial for object detection and has to be performed when environmental conditions change. The calibration procedure should be effective and fast. The proposed calibration algorithm processes the image acquired by the camera and analyzes a white area in the image to calibrate the white-balance, a black area to calibrate the brightness and the histogram of the intensities of the image to calibrate the exposure and gain. The black and white areas are known in advance.

The histogram of the intensities of an image is a representation of the number of times that each intensity value appears in the image. For an image represented using 8 bits per pixel, the possible values are between 0 and 255. Image histograms can indicate if the image is underexposed or overexposed.

The assumptions used by the proposed algorithm are:

- the white area should be white - in the YUV color space this means that the average value of $\mathrm{U}$ and $\mathrm{V}$ should be 127 . If the white-balance is not correctly configured, these values are different from 127 and the image does not have the correct colors;

- the black area should be black - in the RGB color space, this means that the average values of $\mathrm{R}, \mathrm{G}$ and $\mathrm{B}$ should be close to zero. If the brightness is too high, it is observed that the black region becomes blue, resulting in a degradation of the image;

- the distribution of the intensity histogram should be centered around 127 . Dividing the histogram into regions, the left regions represent dark colors, while the right regions represent light colors. An underexposed image will have the histogram be leaning to the left, while an overexposed image will have the histogram leaning to the right (for an example see the Fig. 4 a)).

Statistical measures can be extracted from digital images to quantify the image quality $[10,11]$. A number of typical measures used in the literature can be computed from the image gray level histogram, namely, the mean $(\mu)$, the entropy $(E)$, the absolute central moment $(A C M)$ and the mean sample value $(M S V)$ :

$$
\begin{gathered}
\mu=\sum_{i=0}^{N-1} i P(i) \quad E=-\sum_{i=0}^{N-1} P(i) \log (P(i)) \\
A C M=\sum_{i=0}^{N-1}|i-\mu| P(i) \quad M S V=\frac{\sum_{j=0}^{4}(j+1) x_{j}}{\sum_{j=0}^{4} x_{j}},
\end{gathered}
$$

where $N$ is the number of possible gray values in the histogram (typically, 256), $P(i)$ is the probability of each gray value and $x(j)$ is the sum of the gray values in region $j$ of the histogram (in the proposed approach we divided the histogram into five regions). When the histogram values of an image are uniformly distributed in the possible values, then $\mu \approx 127, E \approx 8, A C M \approx 60$ and 
$M S V \approx 2.5$. In Section 3 we use these measures to analyze the images acquired by the camera and to characterize the performance of the proposed calibration algorithm. Moreover, we use the information of MSV to calibrate the exposure and the gain of the camera.

The algorithm configures the most important parameters of the camera: exposure, gain, white-balance and brightness. For each one of these parameters, a PI controller was implemented. PI controllers are used instead of proportional controllers as they result in better control having no stationary error. The constants of the controller have been obtained experimentally for both cameras, guaranteeing the stability of the system and an acceptable time to reach the desired reference [12].

The algorithm (see Fig. 1) configures one parameter at a time, iterating between them when the convergence of the parameter under analysis has been attained. The algorithm stops when all the parameters have converged. This procedure solves the problem of the correlation that exists between the parameters. After configuring one parameter, the others are configured taking into account the new values.

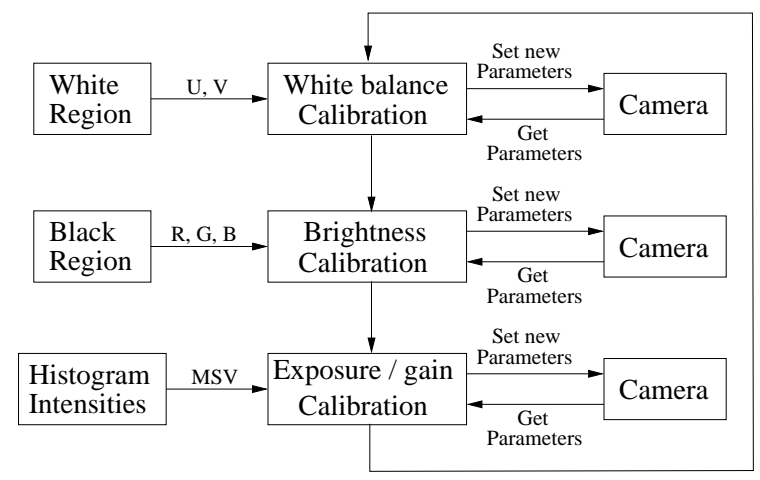

Fig. 1. Overview of the automated calibration procedure. The algorithm executes one module at a time, changing between them when the convergence of the parameter under analysis has been attained.

\section{Experimental results}

To measure the performance of this calibration algorithm, tests have been conducted using the camera with different initial configurations. In Fig. 2, the experimental results are presented both when the algorithm starts with the parameters of the camera set to zero as well as when set to the maximum value. As it can seen, the configuration obtained after using the proposed algorithm is approximately the same, independently of the initial configuration of the cam- 
era. Moreover, the algorithm converges fast (it takes between 60 and 70 frames to converge).
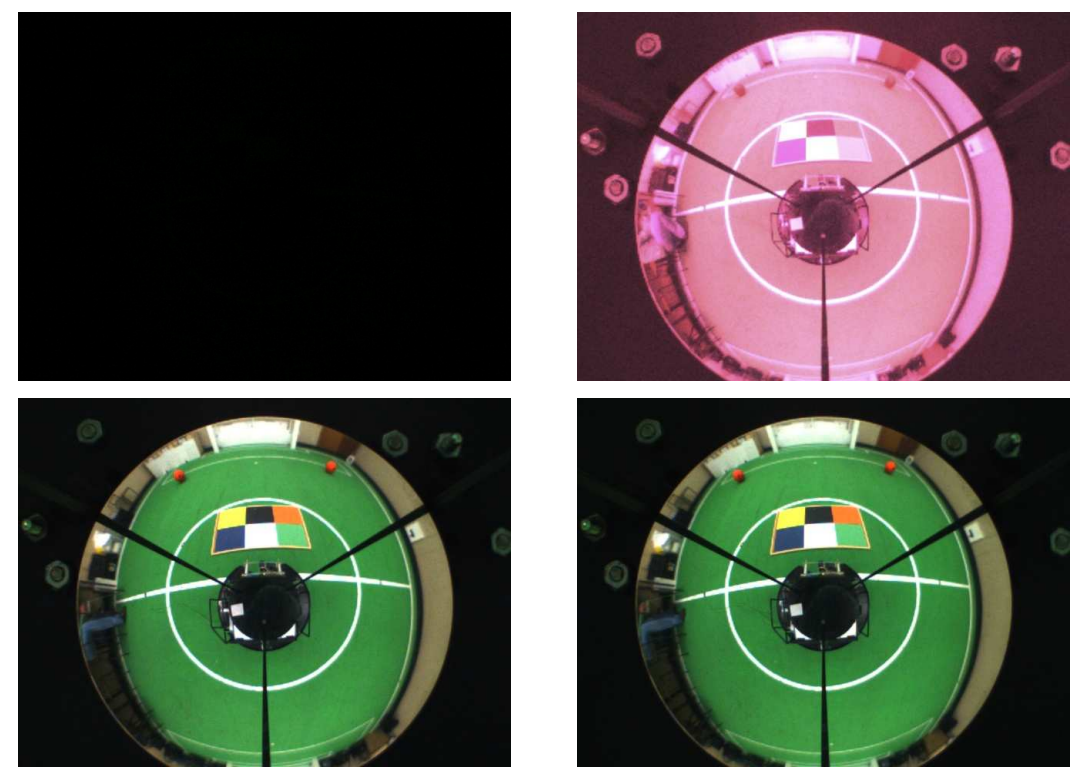

Fig. 2. Some experiments using the automated calibration procedure. On the left, results obtained starting with all the parameters of the camera set to zero. On the right, results obtained with all the parameters set to the maximum value. At the top, the initial image acquired. In the last row, the image obtained after applying the automated calibration procedure.

In Fig. 3 we present an image acquired with the camera in auto mode. The results obtained using the camera with the parameters in auto mode are overexposed and the white balance is not correctly configured. This is due to the fact that the camera analyzes the entire image and, as can be seen in Fig. 2, there are large black regions corresponding to the robot itself. Our approach uses a mask to select the region of interest, in order to calibrate the camera using only the valid pixels. Moreover, and due to the changes in the environment around the robot as it moves, leaving the camera in auto mode leads to undesirable changes in the parameters of the camera, causing problems to the correct color classification.

Table 1 presents the value of the statistical measures described to evaluate the quality of digital images, regarding the experimental results presented in Fig. 2. These results confirm that the camera is correctly configured after applying the automated calibration procedure, since the results obtained are near the optimal. Moreover, independently of the initial configuration, we obtain images with the same characteristics. 

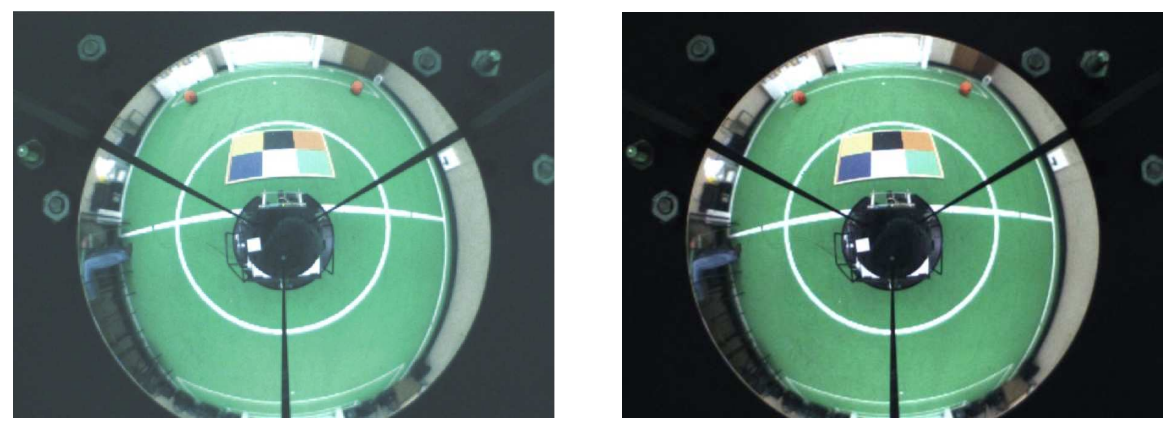

Fig. 3. On the left, an example of an image acquired with the camera parameters in auto mode. On the right, an image acquired after applying the automated calibration algorithm.

Table 1. Statistical measures obtained for the images presented in Figs. 2 and 3. The initial values refer to the images obtained with the camera before applying the proposed automated calibration procedure. The final values refer to the images acquired with the cameras configured with the proposed algorithm.

\begin{tabular}{c|l|r|r|c|c}
\hline Experiment & - & ACM & Average & Entropy & MSV \\
\hline \hline Parameters & Initial & 111.00 & 16.00 & 0.00 & 1.00 \\
\cline { 2 - 6 } set to zero & Final & 39.18 & 101.95 & 6.88 & 2.56 \\
\hline \hline \multirow{2}{*}{$\begin{array}{c}\text { Parameters. } \\
\text { set to maximum }\end{array}$} & Initial & 92.29 & 219.03 & 2.35 & 4.74 \\
\cline { 2 - 6 } & Final & 42.19 & 98.59 & 6.85 & 2.47 \\
\hline \hline Camera in & Initial & 68.22 & 173.73 & 6.87 & 3.88 \\
\cline { 2 - 6 } Auto Mode & Final & 40.00 & 101.14 & 6.85 & 2.54 \\
\hline \hline
\end{tabular}

According to the experimental results presented in Table 1, we conclude that the MSV measure is the best one in classifying the quality of an image. This measure can distinguish between two images that have close characteristics, as the case when the camera is used in auto mode. This observation led us to use it in the calibration procedure.

The good results of the automated calibration procedure can also be confirmed by the histograms presented in Fig. 4. The histogram of the image obtained after applying the proposed automated calibration procedure (Fig. 4b) is centered near the intensity 127 , which is a desirable property, as visually confirmed in Fig. 2. The histogram of the image acquired using the camera in auto mode (Fig. 4a) shows that the image is overexposed, leading to the majority of the pixels to have saturated values.

This algorithm has also been tested outdoors, under natural light. Figure 5 shows that the algorithm works well even with different light conditions. It confirms that the algorithm could be used in non-controlled lighting conditions and under different environments. 


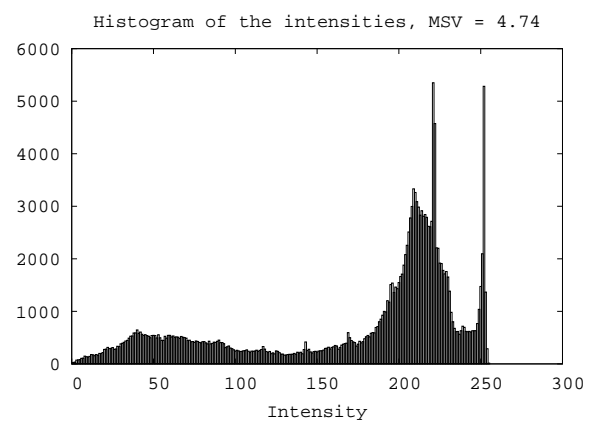

a)

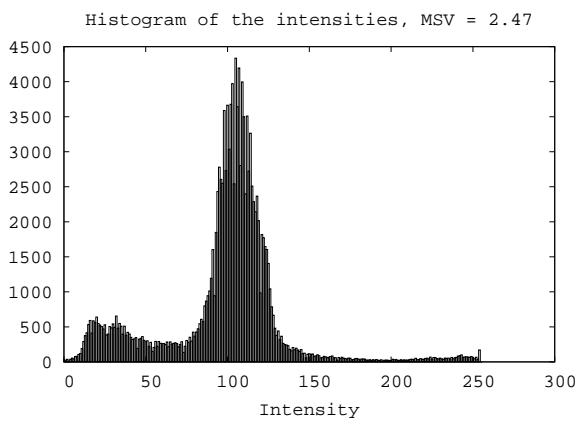

b)

Fig. 4. The histogram of the intensities of the two images presented in Fig. 2. a) shows the histogram of the image obtained with the camera parameters set to maximum. b) shows the histogram of the image obtained after applying the automated calibration procedure.
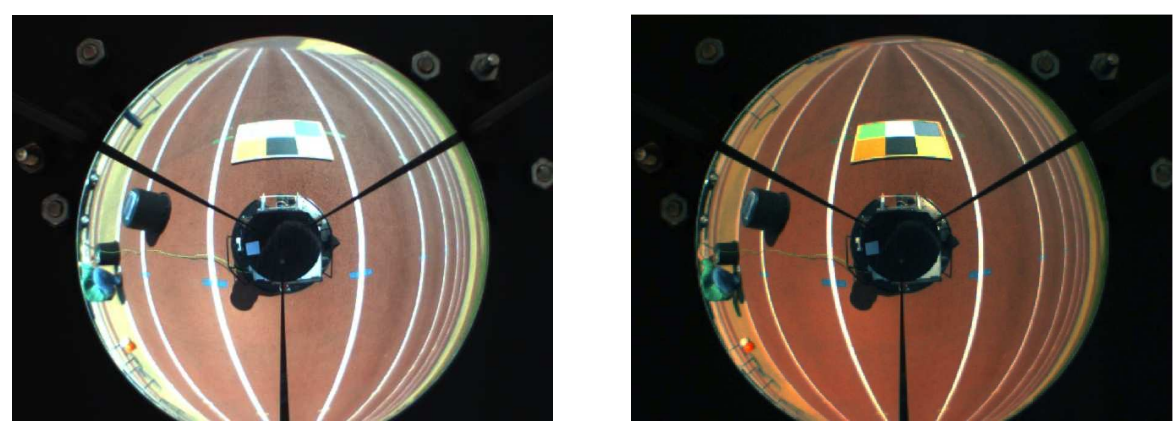

Fig. 5. On the left, an image acquired outdoors using the camera in auto mode. As it is possible to observe, the colors are washed out. That happens because the camera's auto-exposure algorithm tries to compensate the black around the mirror. On the right, the same image with the camera calibrated using the implemented algorithm. As can be seen, the colors and their contours are much more defined.

\section{Conclusions}

We propose an algorithm to autonomously configure the most important parameters of a digital camera. This procedure requires a few seconds for a single robot, which is much faster than the manual calibration performed by an expert user. The experimental results obtained show that the algorithm converges independently of the initial configuration of the camera. Moreover, we have analyzed the images obtained after the proposed calibration algorithm using statistical measures and we concluded that the images have the desired characteristics. These results allow the use of the same color classification independently of the lighting conditions. 
The calibration algorithm is also used in run-time in order to adjust the camera parameters during a game, because the illumination along the field could not be constant and the nature of the light and its intensity could change during a game. This algorithm contributed to success of the CAMBADA team during the RoboCup2008, allowing it to distinctively achieve the 1st place.

\section{References}

1. Mayer, G., Utz, H., Kraetzschmar, G.: Playing robot soccer under natural light: A case study. In: Proc. of the RoboCup 2003. Volume 3020 of Lecture Notes on Artificial Inteligence., Springer (2003)

2. Heinemann, P., Sehnke, F., S., F., Zell, A.: Towards a calibration-free robot: The act algorithm for automatic online color training. (2007) 363-370

3. Hafner, R., Lange, S., Lauer, M., Riedmiller, M.: Brainstormers tribots team description. Technical report, Institute of Computer Science, Institute of Cognitive Science (2008) University of Osnabru, Germany.

4. Zhang, H., Lu, H., Wang, X., Sun, F., Ji, X., Hai, D., Liu, F., Cui, L., Zheng, Z.: Nubot team description paper 2008. Technical report, College of Mechatronics and Automation (2008) National University of Defense Technology, China.

5. Lunenburg, J.J.M., v.d. Ven, G.: Tech united team description. Technical report, Control Systems Technology Group (2008) Eindhoven University of Technology, Netherlands.

6. Bouchard, B., Lapensée, D., Lauzon, M., Pelletier-Thibault, S., Roy, J.C., Scott, G.: Robofoot Épm team description paper 2008. Technical report, Mechatronics Laboratory (2008) École Polytechnique de Montréal, Canada.

7. Krawczyk, G., Goesele, M., Seidel, H.: Photometric calibration of high dynamic range cameras. Research Report MPI-I-2005-4-005, Max-Planck-Institut für Informatik, Stuhlsatzenhausweg 85, 66123 Saarbrücken, Germany (April 2005)

8. Azevedo, J.L., Lau, N., Corrente, G., Neves, A., Cunha, M.B., Santos, F., Pereira, A., Almeida, L., Lopes, L.S., Pedreiras, P., Vieira, J., Martins, D.A., Figueiredo, N., Silva, J., Filipe, N., Pinheiro, I.: CAMBADA'2008: Team description paper. Technical report, Universidade de Aveiro, Portugal (2008)

9. Neves, A.J.R., Martins, D.A., Pinho, A.J.: A hybrid vision system for soccer robots using radial search lines. In: Proc. of the 8th Conference on Autonomous Robot Systems and Competitions, Portuguese Robotics Open - ROBOTICA'2008, Aveiro, Portugal (April 2008) 51-55

10. Shirvaikar, M.V.: An optimal measure for camera focus and exposure. In: Proc. of the IEEE Southeastern Symposium on System Theory, Atlanta, USA (March 2004)

11. Nourani-Vatani, N., Roberts, J.: Automatic camera exposure control. In: Proc. of the 2007 Australasian Conference on Robotics and Automation, Brisbane, Australia (December 2007)

12. D’Azzo, J.J., Houpins, C.H., Sheldon, S.N.: Linear Control System Analysis and Design with Matlab. CRC Press (2003) 